\title{
How Larger Society Can Give a Helping Hand to Worldwide FINGERS
}

\section{T. Daly}

Sorbonne Université, Sciences Norms Democracy, UMR 8011, Paris, France.

Corresponding Author: Timothy Daly, Sorbonne Université, Sciences Norms Democracy, UMR 8011, Paris, France, timothy.daly@sorbonne-universite.fr

\section{Dear Editor,}

A s Rosenberg and colleagues describe in the journal (1), the Worldwide FINGERS is "a landmark initiative" to develop "Multidomain Interventions to Prevent Cognitive Impairment, Alzheimer's Disease, and Dementia" across the globe (p. 35, ibid). It demonstrates the research community's investment to move beyond pharmacological-only approaches for these conditions, and to find actionable solutions across the high/low income country divide "with shared core methodology, but local culture and context-specific adaptations" (p. 29, ibid). There are four steps that larger society, including governments and public health bodies, could take now to bolster dementia risk reduction efforts.

Firstly, help individuals and communities carry out a FINGER-inspired lifestyle that involves physical, mental, and social stimulation, nutritional changes, and management of metabolic and vascular risk factors. Existing government campaigns have recommended lifestyle changes (2), but each recommendation ought to be accompanied by investment in making it feasible, e.g. by "ensuring people have access to affordable, healthy food, rather than just encouraging them to eat healthily" (3).

Secondly, take action against social determinants of health. Rosenberg and colleagues recognise the importance of economic development for dementia. The wealth-brain health link is a double-edged sword: socioeconomic deprivation not only increases the risk of dementia but also reduces participation in FINGERstyle interventions (4). It is therefore important that research with these interventions is complemented by action against the social determinants of health such as socioeconomic deprivation, barriers to education, and social isolation as three particularly relevant examples.
Thirdly, not be moralistic about participation in riskreduction activities. The data on the impact of these interventions on individuals is still not definitive. In a pluralistic society, it is important to respect those who do not take part and to warn against victim-blaming where people with dementia are seen as complicit for not having done more to prevent their condition (2).

Finally, design a risk-reducing society. Living and working spaces ought to be made more conducive to encouraging participation in risk-reduction activities. This means making those environments not only safe and accessible, but also as physically, mentally, and socially stimulating as possible across society (5).

In conclusion, the vital Worldwide FINGERS initiative would be greatly helped along by the further collaboration of broader society to build a positive and democratic view of dementia in which risk reduction is seen as a shared challenge and responsibility.

Acknowledgements: Timothy Daly thanks the Fondation Médéric Alzheimer for the doctoral bursary that funds his studies.

Conlict of interest: The author declares no conflicts of interest.

\section{References}

1. A. Rosenberg, F. Mangialasche, T. Ngandu, A. Solomon, M. Kivipelto, Multidomain Interventions to Prevent Cognitive Impairment, Alzheimer's Disease, and Dementia: From FINGER to World-Wide FINGERS. J Prev Alzheimers Dis 2020;7, 29-36, doi: 10.14283/jpad.2019.41.

2. D. Horstkötter, K. Deckers, S. Köhler, Primary Prevention of Dementia: An Ethical Review. J Alzheimers Dis 2021;79, 467-476, doi: 10.3233/JAD-201104.

3. The Lancet Neurology, Amid competing priorities, dementia must not be forgotten. Lancet Neurol 2021;20, 685, doi: 10.1016/S1474-4422(21)00258-1.

4. N. Coley et al., Disparities in the participation and adherence of older adults in lifestyle-based multidomain dementia prevention and the motivational role of perceived disease risk and intervention benefits: an observational ancillary study to a randomised controlled trial. Alzheimers Res Ther 2021;13, 157, doi: 10.1186/s13195-021-00904-6.

5. T. Daly, Giving a fairer face to urban space: Progress on the long road to dementia prevention. Int J Geriatr Psychiatry, 2021, doi: 10.1002/ gps.5657.

How to cite this article: T. Daly. How Larger Society Can Give a Helping Hand to Worldwide FINGERS. J Prev Alz Dis 2022;1(9):185; http://dx.doi. org/10.14283/jpad.2022.11 